\title{
SUPERCRITICAL CO, RECOVERY OF CAFFEINE FROM GREEN COFFEE OIL: NEW EXPERIMENTAL SOLUBILITY DATA AND MODELING
} Álvaro Bandeira Antunes de Azevedo, Theo Guenter Kieckbusch, Alexandre Keiji Tashima and Rahoma Sadeg
Mohamed $^{+}$

Faculdade de Engenharia Química, Universidade Estadual de Campinas, CP 6066, 13083-970 Campinas - SP, Brasil

Paulo Mazzafera*

Instituto de Biologia, Universidade Estadual de Campinas, CP 6109, 13083-970 Campinas - SP, Brasil

Silvio Alexandre Beisl Vieira de Melo

Departamento de Engenharia Química, Escola Politécnica de Engenharia, Universidade Federal da Bahia, 40210-630 Salvador - BA, Brasil

Recebido em 23/4/07; aceito em 17/12/07; publicado na web em 13/8/08

\begin{abstract}
The caffeine solubility in supercritical $\mathrm{CO}_{2}$ was studied by assessing the effects of pressure and temperature on the extraction of green coffee oil (GCO). The Peng-Robinson ${ }^{1}$ equation of state was used to correlate the solubility of caffeine with a thermodynamic model and two mixing rules were evaluated: the classical mixing rule of van der Waals with two adjustable parameters (PR-VDW) and a density dependent one, proposed by Mohamed and Holder $^{2}$ with two (PR-MH, two parameters adjusted to the attractive term) and three (PR-MH3 two parameters adjusted to the attractive and one to the repulsive term) adjustable parameters. The best results were obtained with the mixing rule of Mohamed and Holder $^{2}$ with three parameters.
\end{abstract}

Keywords: caffeine solubility; coffee oil; supercritical carbon dioxide.

\section{INTRODUCTION}

Several reports in the literature describe the solubility of caffeine in supercritical $\mathrm{CO}_{2}$ and supercritical $\mathrm{CO}_{2}$ plus entrainers (co-solvents), such as ethanol, water, isopropanol, etc. ${ }^{3-8}$ Reports on studies of the solubility of caffeine regarding its recovery from green coffee oil, however, are not disclosed in the literature. Therefore, the objective of this work was to obtain new experimental data on caffeine solubility in supercritical $\mathrm{CO}_{2}$ to assess the effects of thermodynamic variables (pressure and temperature) on the extraction of green coffee oil (GCO). The Peng-Robinson equation of state ${ }^{1}$ was used to correlate the solubility data for caffeine- $\mathrm{CO}_{2}$ system with a thermodynamic model. Two mixing rules were evaluated: the classical mixing rule of van der Waals with two adjustable parameters (PR-VDW) and a density dependent one, proposed by Mohamed and Holder $^{2}$ with two (PR-MH, two parameters adjusted to the attractive term) and three (PR-MH3 two parameters adjusted to the attractive term and one to the repulsive term) fitting parameters.

\section{EXPERIMENTAL}

\section{Materials}

Pure carbon dioxide (99.9\%) was obtained from White Martins Inc. (Campinas, Brazil). Caffeine (99.9\%) was purchased from Sigma (St. Louis, USA).

\section{Experimental apparatus}

A semi-continuous flow experimental apparatus (Figure 1) with independent control of temperature and pressure was used in the extraction experiments. The apparatus was projected and assembled by the supercri-

\footnotetext{
${ }^{\dagger}$ In Memoriam

*e-mail:pmazza@unicamp.br
}

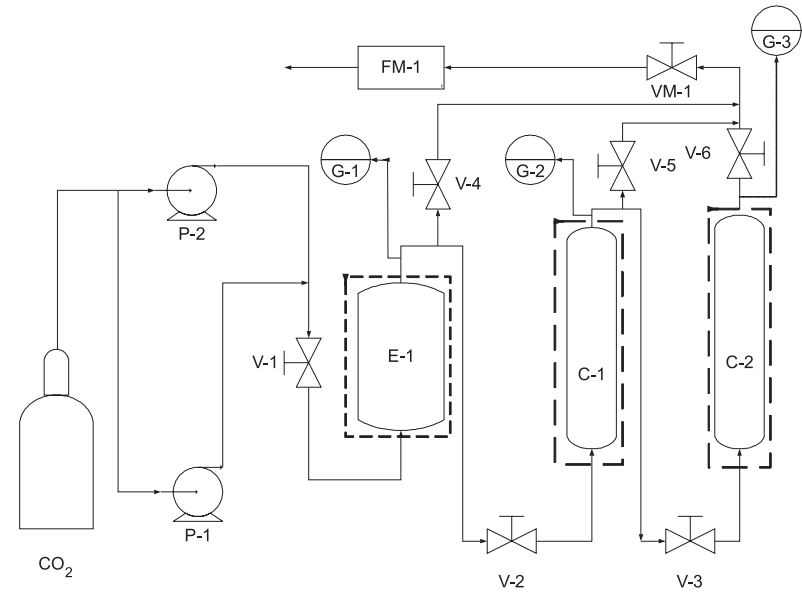

Figure 1. Experimental apparatus process diagram

tical fluid process research group of the School of Chemical Engineering, State University of Campinas. The apparatus was designed for pressures up to $41.3 \mathrm{MPa}$ at $200{ }^{\circ} \mathrm{C}$. The major components of this apparatus are positive liquid displacement pumps (P-1 and P-2, Thermal Separation Products, Riveira Beach, FL, USA) for solvent delivery (46-460 mL/h), one $300 \mathrm{~mL}$ high-pressure extraction vessel (E-1, Autoclave Engineers, Erie, PA), two high-pressure columns (C-1 and C-2, 300 mm X 12.7 $\mathrm{mm}$ I.D.) and collection flasks. The extraction vessel and the columns were supplied with heating jackets and temperature controllers and were operated in series. Heating tapes were used throughout the equipment to maintain constant temperature over the entire apparatus. In order to ensure constant and steady solvent delivery, the pump heads were cooled by a circulating fluid passing through a chiller, CFT R134a (Neslab Instruments, Newington, NH, USA). Flow rates and accumulated gas volumes passing through the apparatus were measured using a digital flow meter device (FM-1, EG\&G Instruments Flow Technology, Gai- 
thersburg, MD, USA). Autoclave Engineers (Erie, PA, USA) micro metering valves (VM-1) were used for flow control throughout the apparatus. Heating tapes were also used around these valves to prevent freezing following depressurization. Pressure in extractor and columns were monitored with a digital transducer system (G1, G2 and G3, Heise Series 901A RTS acquired from Dresser Industries, Stratford, CT, USA) with a precision of $\pm 0.03 \mathrm{MPa}$. Extractor and columns temperatures were controlled to $\pm 0.5^{\circ} \mathrm{C}$.

\section{Experimental procedures}

To asses the reliability of the experimental apparatus and to validate the experimental data obtained with this equipment, two caffeine solubility experiments were performed and the results compared with previous data from Johannsen and Brunner. ${ }^{9}$

To determine the caffeine solubility in supercritical $\mathrm{CO}_{2}$ experimental runs were carried out at temperatures of 50,60 and 70 ${ }^{\circ} \mathrm{C}$ and pressures of 15.2 to $35.2 \mathrm{MPa}$. Approximately $30 \mathrm{~g}$ of pure caffeine were feed to the extractor mixed with glass beads to improve the mass transfer and solvent distribution due to an increase of the contact area between solute and solvent. The solvent was fed slowly to the extractor and when the pre-determined operation conditions were attained the experimental solubility data were obtained through the dynamic method with continuous flow. The flow rate of $\mathrm{CO}_{2}$ was fixed in $1 \mathrm{~L} / \mathrm{min}$ of the expanded gas. This value was established based on the work of Kopcak and Mohamed, ${ }^{5}$ who determined the caffeine solubility in supercritical solvents $\left(\mathrm{CO}_{2} /\right.$ ethanol 5\% w/w and $\mathrm{CO}_{2}$ /isopropanol 5\%, w/w). Samples were collected after $30 \mathrm{~g}$ of $\mathrm{CO}_{2}$ have passed through the extractor, and each point represents the average value of six independent experimental determinations. Samples were collected by precipitation of caffeine after depressurization over a micrometric valve. The $\mathrm{CO}_{2}$ masses were determined using a flow computer measuring device. The caffeine solubility data were calculated in a molar basis. The amount of caffeine in the samples was determined by dissolving it in ethanol $(250 \mathrm{~mL})$, and $1 \mathrm{~mL}$ was sampled and further diluted to $100 \mathrm{~mL}$. The absorbance of this solution was obtained at $277 \mathrm{~nm}$ and compared with a standard curve obtained with pure caffeine solutions at several concentrations.

The experimental assays of caffeine extraction from green coffee oil (GCO) were carried out feeding $10 \mathrm{~g}$ of green coffee oil mixed with glass beads. Caffeine content in this oil was $2.7 \mathrm{~g} / \mathrm{kg}$. The assays used the continuous flow dynamic method over the same range of temperature $\left(50\right.$ to $\left.70{ }^{\circ} \mathrm{C}\right)$ and pressure $(15.2$ to $35.2 \mathrm{MPa})$ used in the pure caffeine solubility determinations. Caffeine in the fractions collected were determined by HPLC, using a UV SDP-10AV Shimadzu operating at $280 \mathrm{~nm}$ and a C18 column $(4.6 \mathrm{~mm}$ x $250 \mathrm{~mm}, 5$ $\mu \mathrm{m}$, Supelco). The mobile phase was an aqueous solution of methanol $(50 \%)$ and acetic acid $(0.5 \%)$ at a flow rate of $0.8 \mathrm{~mL} / \mathrm{min}$. Pure caffeine was used to build a calibration curve. The caffeine solubility was calculated in mass and molar basis.

After each determination the experimental apparatus was washed with ethanol until the caffeine concentration in the solvent was in the error range. After the washing the equipment was dried with a flow of $\mathrm{CO}_{2}$.

\section{Solubility correlations}

The caffeine solubility was calculated considering the supercritical phase as a compressed gas and caffeine as a pure solid phase, according to McHugh and Krukonis, ${ }^{10}$ resulting in the following equation:
$y_{i}=\frac{P_{i}^{s u b}(T) \exp \left[\frac{P v_{i}^{s}}{R T}\right]}{\phi_{i}^{S C} P}$

where $P_{i}^{s u b}(T)$ is the caffeine sublimation pressure at temperature $T$, $P$ is the equilibrium pressure, $v_{i}^{S}$ is the solid molar volume, $R$ is the universal gas constant and $\phi_{i}^{\mathrm{SC}}$ is the solute fugacity coefficient in the supercritical phase. $\phi_{i}^{\mathrm{SC}}$ was calculated using the Peng-Robinson ${ }^{1}$ cubic equation of state, given by the following expression:

$P=\frac{R T}{v-b}-\frac{a}{v(v+b)+b(v-b)}$

The parameters $a$ (attractive term) and $b$ (repulsive term) represent the attraction force among the molecules and their volumes, respectively. In multi-component systems, $a$ and $b$ are functions of composition, and mixing rules are employed. In the classical mixing rule of van der Waals, one interaction parameter for $a$ and one for $b$ are adjusted to the experimental data, resulting in the expressions:

$$
\begin{aligned}
& a=\sum_{i} \sum_{j} x_{i} x_{j} a_{i j} \\
& a_{i j}=\sqrt{a_{i} a_{j}}\left(1-\delta_{i j}\right) \\
& b=\sum_{i} \sum_{j} x_{i} x_{j} b_{i j} \\
& b_{i j}=\frac{b_{i}+b_{j}}{2}\left(1-\eta_{i j}\right)
\end{aligned}
$$

where $\delta_{i j}$ and $\eta_{i j}$ are the interaction parameters. One disadvantage of the classical mixing rule is that one set of parameters have to be fit at each temperature. An alternative is to turn the adjustable parameters density dependent, and therefore requiring the adjustment of only one set which takes effects of temperature and pressure variations simultaneously into account, as proposed by Mohamed and Holder. ${ }^{2}$ The authors considered a linear variation of the parameter $\delta_{i j}$ with the density $(\rho)$ :

$\delta_{i j}=\alpha_{i j}+\beta_{i j} \rho$

where $\alpha_{i j}$ and $\beta_{i j}$ are adjustable parameters. The mixing rule of Mohamed and Holder ${ }^{2}$ converts the Peng-Robinson ${ }^{1}$ equation in a quartic order in relation to volume. The algorithm of the thermodynamic models were previously tested with the solubility data of naproxen in $\mathrm{CO}_{2}$ from Ting et al.,${ }^{11}$ resulting in the same interaction parameters found by these authors who used the classical one parameter mixing rule.

Caffeine critical properties were obtained using Joback group contribution correlations and an experimental value of the boiling temperature previously reported in the literature $\left(\mathrm{T}_{\mathrm{b}}=628 \mathrm{~K}\right)$. The sublimation vapor pressures of caffeine were obtained using the correlation of Bothe and Cammenga: ${ }^{12}$

$$
\log P^{s u b}(P a)=-\left(\frac{A}{T}\right)+B
$$

The parameters of the Equation $(A=5781 \mathrm{~K}$ and $\mathrm{B}=15.031)$ were obtained by regression of experimental data of caffeine sublimation pressure over the temperature range of 350 to $509 \mathrm{~K}$. 


\section{RESULTS AND DISCUSSION}

\section{Caffeine solubility in carbon dioxide}

The solubility data obtained for caffeine in pure $\mathrm{CO}_{2}$ are shown in terms of mole fraction in Figure 2, and correspond to the mean of six independent experimental runs with an average error of $6 \%$. The data obtained at $60^{\circ} \mathrm{C}$ are in good agreement with the previous values reported by Johannsen and Brunner, ${ }^{9}$ validating the experimental methodology and measurements in the apparatus. Table 1 presents the solubility data converted to mass fraction.

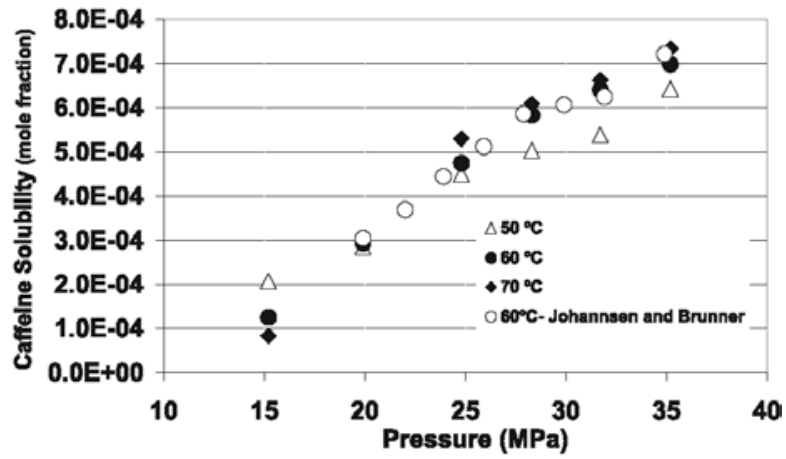

Figure 2. Caffeine solubility in supercritical carbon dioxide at $50{ }^{\circ} \mathrm{C}(\Delta)$, $60{ }^{\circ} \mathrm{C}(\bullet)$ and $70{ }^{\circ} \mathrm{C}(\bullet)$. Data of Johannsen and Brunner 9 at $60{ }^{\circ} \mathrm{C}\left({ }^{\circ}\right)$

Table 1. Caffeine solubility in supercritical $\mathrm{CO}_{2}(\mathrm{~g} / \mathrm{g})$

\begin{tabular}{lccc}
\hline Pressure (MPa) & \multicolumn{3}{c}{ Caffeine Solubility $(\mathrm{g} / \mathrm{g})$} \\
& $50{ }^{\circ} \mathrm{C}$ & $60{ }^{\circ} \mathrm{C}$ & $70{ }^{\circ} \mathrm{C}$ \\
\hline 15.2 & $7.75 \mathrm{E}-04$ & $4.72 \mathrm{E}-04$ & $2.36 \mathrm{E}-04$ \\
19.9 & $1.07 \mathrm{E}-03$ & $1.11 \mathrm{E}-03$ & $1.13 \mathrm{E}-03$ \\
24.8 & $1.67 \mathrm{E}-03$ & $1.79 \mathrm{E}-03$ & $1.98 \mathrm{E}-03$ \\
28.3 & $1.88 \mathrm{E}-03$ & $2.21 \mathrm{E}-03$ & $2.27 \mathrm{E}-03$ \\
31.7 & $2.01 \mathrm{E}-03$ & $2.42 \mathrm{E}-03$ & $2.47 \mathrm{E}-03$ \\
35.2 & $2.40 \mathrm{E}-03$ & $2.64 \mathrm{E}-03$ & $2.74 \mathrm{E}-03$ \\
\hline
\end{tabular}

The solubility data also show that increasing pressure at constant temperature increases caffeine solubility in supercritical $\mathrm{CO}_{2}$ due to the increase of solvent density and consequently the solvation power. This tendency can also be associated to an increase in the magnitude of the physical intermolecular interactions between the solvent and solute molecules, since these are short range forces that strengthen as the distance decreases. ${ }^{13-15}$

The temperature effect on the solubility shows the occurrence of a retrograde behavior at pressures lower than 19.3 MPa. Below this level of pressure, known as crossover pressure, the caffeine solubility decreases as temperature increases. This behavior is a consequence of the solvent density and the solute vapor pressure variation effect on solubility. ${ }^{16,17}$ Near the critical point, below the crossover pressure small increases in temperature result in drastic decrease in solvent density and consequently, on its solvent capability. As the process pressure moves away from the solvent crossover pressure its density become less sensitive to temperature changes and the effect that prevails is the increase in solute fugacity with temperature resulting in a solute solubility increase.

The retrograde behavior observed at the lower pressure levels is commonly found in supercritical extraction processes. The results obtained with caffeine are very close to values for crossover pressure found in extraction of cupuaçu fat using supercritical $\mathrm{CO}_{2}{ }^{7}{ }^{7,18,19}$

\section{Caffeine extraction from green coffee oil}

Figures $3 \mathrm{a}, \mathrm{b}$ and $\mathrm{c}$ show the caffeine extraction curves obtained at different temperatures and pressures. Most of the caffeine present in GCO is extracted in the first three or four collected fractions. Regarding the fractionation efficiency (preferential removal of caffeine from GCO) of GCO and caffeine, the highest selectivity was observed at $15.2 \mathrm{MPa}$ and $70{ }^{\circ} \mathrm{C}$, since under these conditions, $73 \%$ of the caffeine was removed from GCO with only $6 \%$ of the lipid mass extracted. ${ }^{20}$

Comparing the caffeine extracted per mass of solvent, calculated using the slope of the initial linear portion of the extraction curve (Figures $3 a, b$ and c), it can be seen that the extraction of caffeine from coffee oil is more than one order of magnitude lower than the thermodynamic solubility as given in Table 1 . This can be attributed to mass transfer hindrances and, mainly, to the lower fugacity of the caffeine dissolved in the oil phase. The results of caffeine extraction also indicate that
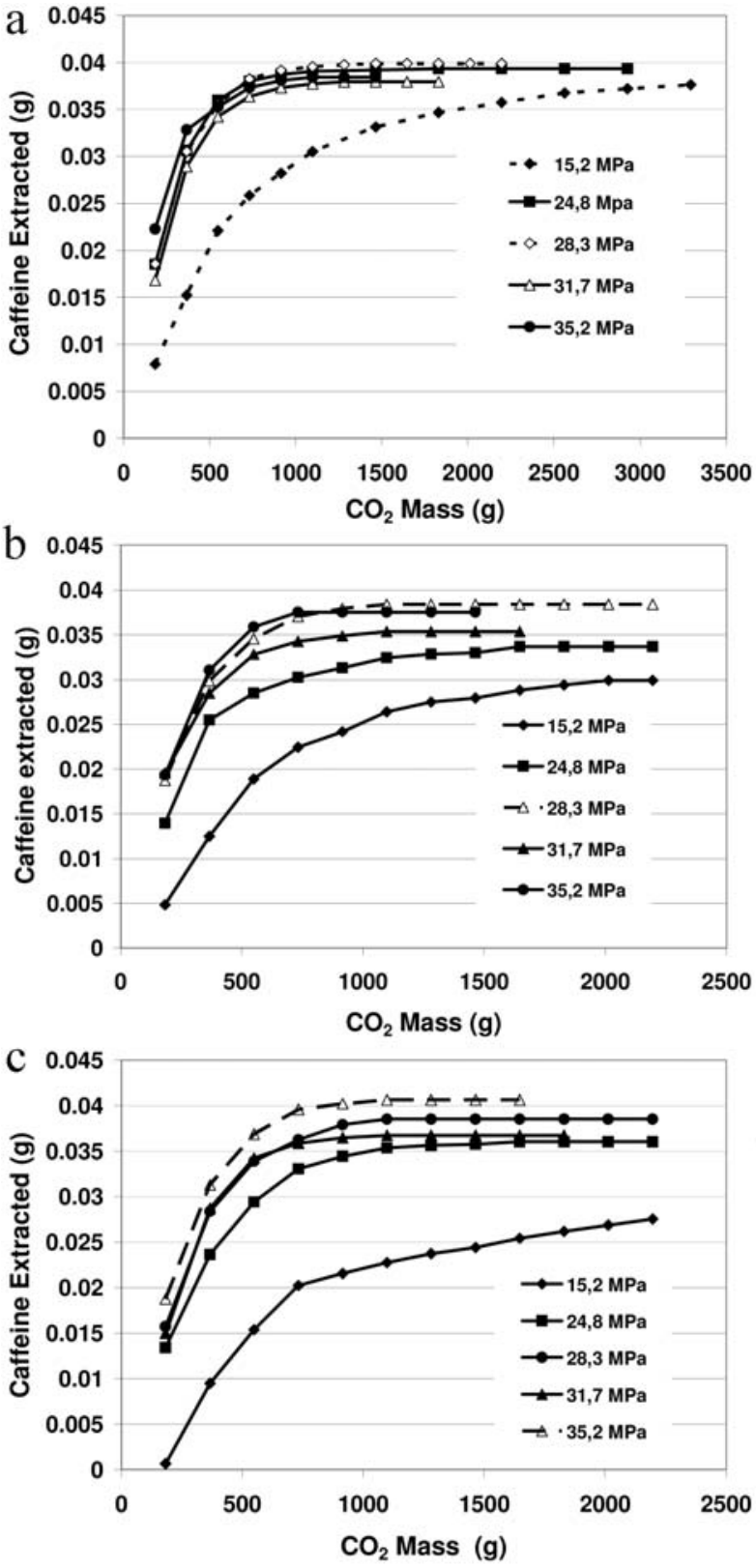

Figure 3. Caffeine extraction curves of green coffee oil at: A) $50{ }^{\circ} \mathrm{C}, \mathrm{B}$ $60^{\circ} \mathrm{C}$ and $\left.\mathrm{C}\right) 70^{\circ} \mathrm{C}$ 
supercritical $\mathrm{CO}_{2}$, even at low-pressure levels exhibits a higher affinity for the triacylglycerols (TAG) in GCO than for caffeine. This could be anticipated since the order of magnitude of pure TAGs solubility data in supercritical $\mathrm{CO}_{2}$ reported by Nilsson et al..$^{21}$ and Neves ${ }^{22}$ fall in the same range of magnitude of the solubility data for coffee oil $\left(10^{-3}-10^{-}\right.$ $\left.{ }^{2}\right) .{ }^{20}$ Considering the chemical nature and physicochemical properties of $\mathrm{CO}_{2}$, it can be regarded as a better solvent for non-polar compounds than for polar ones (e.g. caffeine). ${ }^{23,24}$

The effect of pressure on GCO extraction showed the same trend as observed for caffeine extraction. However above $28 \mathrm{MPa}$, the effect of pressure on the caffeine extraction levels off as can be verified in Figure 3. This can be attributed to synergic effects between the lipids and caffeine as reported by Vasapollo et al. ${ }^{25}$ for the extraction of licopene from dry tomato powder using a vegetable oil as a cosolvent. Therefore as the concentration of co-solvent increases the co-solvent effect diminishes.

The effect of temperature on caffeine extraction can be better appreciated in Figure 4. A retrograde behavior was observed over the pressure range of 15.2 to $28.3 \mathrm{MPa}$. The crossover pressure observed for caffeine in the three component system coffee oil-caffeine- $\mathrm{CO}_{2}$ (approximately $31.7 \mathrm{MPa}$ ) is higher than the crossover pressure in caffeine- $\mathrm{CO}_{2}$ binary system (19.3 MPa). An increase in the cross over pressure is normally detected in solubility isotherms obtained using supercritical solvents modified with a co-solvent due to the increase in the critical temperature and pressure of the mixed solvent. ${ }^{26}$ These authors observed that varying the concentration of ethanol in supercritical $\mathrm{CO}_{2}$ from 0 to $10 \%$ resulted in an increase in the crossover pressure of monocrotaline solubility isotherms from 7.5 to $27.5 \mathrm{MPa}$.

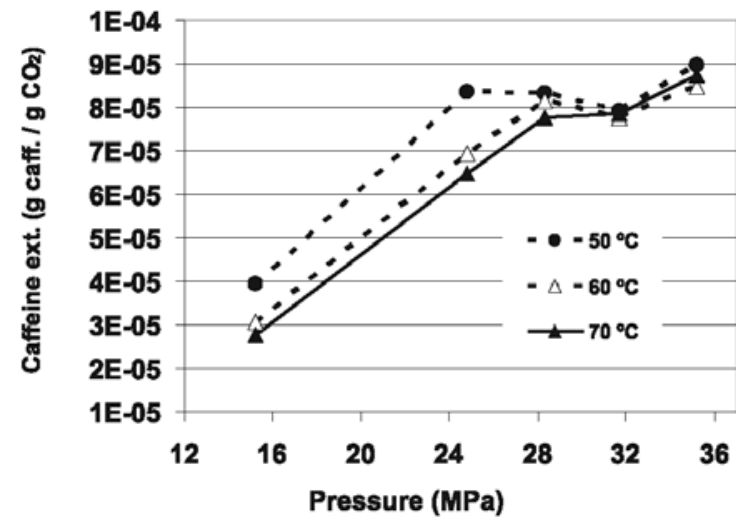

Figure 4. Caffeine equilibrium concentration in the supercritical phase in coffee oil-caffeine $\mathrm{CO}_{2}$ system

The shape of the extraction ratio isotherms in Figure 4 suggest an inflexion point at pressures around $28 \mathrm{MPa}$. This behavior cannot be foreseen by thermodynamics reasonings, since solubility should steadily increase as pressure increases. Up to about $28 \mathrm{MPa}$ the caffeine extraction ratio increases sharply with pressure due the strong variations of the in the vicinity of critical point. This slope diminishes as pressure assumes higher values when the density of the supercritical phase approaches the liquid phase density. The inflection plateaus between the 24 and $32 \mathrm{MPa}$ were attributed to co-solvent effects of the lipid compounds and low caffeine concentration present in the system which reduce the differences in caffeine extraction ratios.

\section{Solubility correlations}

The experimental data and the calculated solubility curves obtained using the mixing rule presented by Equations 3 to 7 are shown in Figures 5a, b and c.
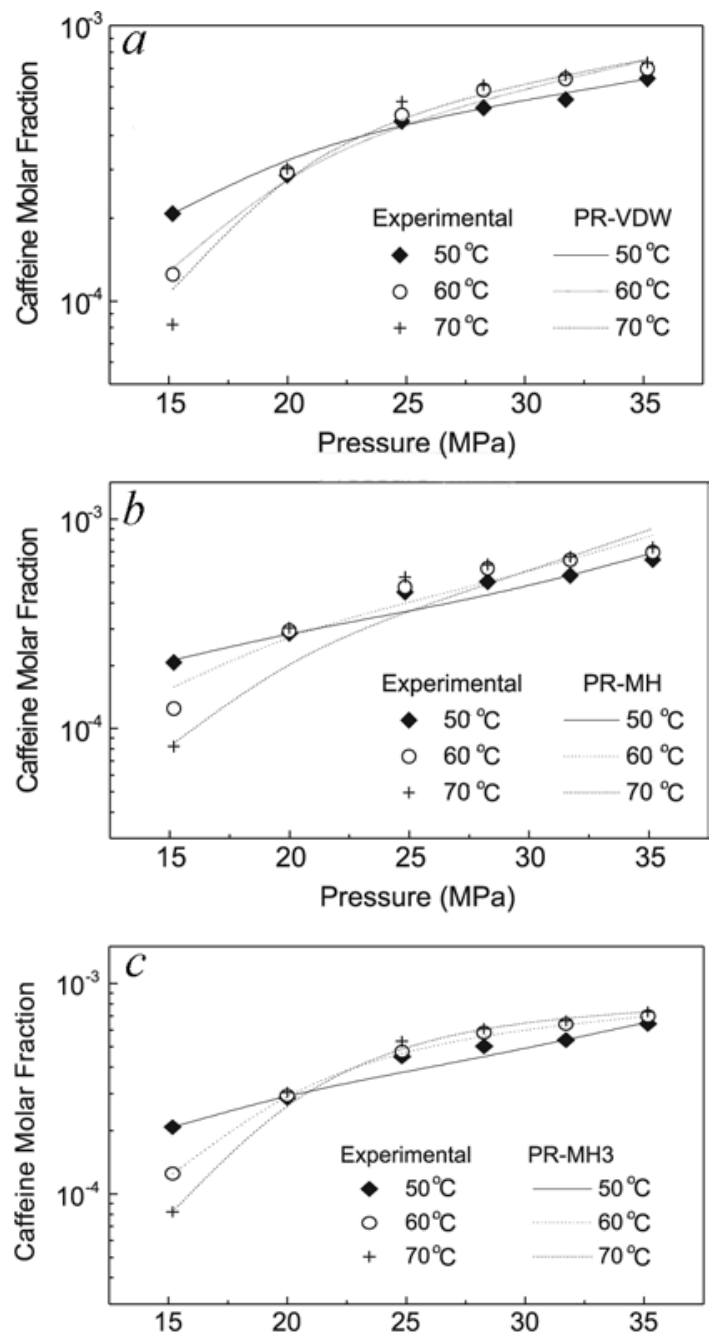

Figure 5. Correlation of caffeine solubility as function of pressure, using (a) the two parameters van der Waals mixing rule $(P R-V D W) ;(b)$ the two parameters Mohamed and Holder mixing rule (PR-MH); (c) using the three parameters Mohamed and Holder ${ }^{2}$ mixing rule (PR-MH3)

The interaction parameters found by regression of the experimental data using the two parameters van der Waals mixing rule for each isotherm $\left(50,60\right.$ and $\left.70{ }^{\circ} \mathrm{C}\right)$ are shown in Table 2. For the mixing rule of Mohamed and Holder ${ }^{2}$ with two and three parameters, the results are shown in Table 3. Although only one set of parameters would suffice the adjustment for the whole range of temperatures, a separated set for each temperature was obtained in order to compare the average errors with those generated by the classical van der Waals mixing rule.

The results show that when only two parameters are used with both mixing rules, the van der Waals approach gives a better fitting, with lower average errors. This can be attributed to the fact that both parameters of the Mohamed and Holder ${ }^{2}$ mixing rule are located at the attractive term, while in the van der Waals, one parameter in the

Table 2. Interactions parameter for the system caffeine- $\mathrm{CO}_{2}$ with Peng-Robinson equation and the van de Waals mixing rule

\begin{tabular}{lccc}
\hline $\mathrm{T}\left({ }^{\circ} \mathrm{C}\right)$ & $\delta_{\mathrm{ij}}$ & $\eta_{\mathrm{ij}}$ & Av. Error $(\%)$ \\
\hline 50 & 0.834 & 1.803 & 4.4 \\
60 & -0.744 & -1.6703 & 4.7 \\
70 & -0.771 & -1.805 & 9.2 \\
\hline
\end{tabular}


Table 3. Interaction parameters for the system caffeine- $\mathrm{CO}_{2}$ with Peng-Robinson equation and the mixing rule of Mohamed and Holder

\begin{tabular}{lcccc}
\hline \multicolumn{5}{c}{ PR-MH 2 parameters } \\
$\mathrm{T}\left({ }^{\circ} \mathrm{C}\right)$ & $\alpha_{\mathrm{ij}}$ & $\beta_{\mathrm{ij}}$ & $\eta_{\mathrm{ij}}$ & Av. Error \\
$(\%)$ & & & & \\
\hline 50 & -0.825 & 92.028 & - & 7.2 \\
60 & -0.712 & 84.574 & - & 13.5 \\
70 & -0.555 & 71.808 & - & 17.6 \\
\hline \multicolumn{5}{c}{ PR-MH 3 parameters } \\
$\mathrm{T}\left({ }^{\circ} \mathrm{C}\right)$ & $\alpha_{\mathrm{ij}}$ & $\beta_{\mathrm{ij}}$ & $\eta_{\mathrm{ij}}$ & Av. Error \\
$(\%)$ & & \multicolumn{5}{c}{} \\
\hline 50 & -0.827 & 63.584 & -0.553 & 5.3 \\
60 & -0.798 & 46.551 & -0.273 & 2.0 \\
70 & -0.824 & 63.266 & -0.313 & 1.9 \\
\hline
\end{tabular}

attractive term and the other in the repulsive term. When the three Mohamed and Holder $^{2}$ parameter mixing rule was used, excellent fitting was obtained, since the third parameter represents a repulsive term.

\section{CONCLUSIONS}

Extraction runs of caffeine from green coffee oil were conducted, using supercritical $\mathrm{CO}_{2}$ and the results compared to the equilibrium solubility of pure caffeine. The $\mathrm{CO}_{2}$ extracted less then $10 \%$ of its caffeine saturation concentration. The $\mathrm{CO}_{2}$ showed strong affinity to the vegetable oil and the TAG solubilized acted as co-solvent for the caffeine extraction, changing the cross-over pressure of the system and affecting the extraction rate.

The Peng-Robinson ${ }^{1}$ equation of state with two different mixing rules was used to correlate the solubility of pure caffeine. The best fitting was obtained with the three parameters mixing rule of Mohamed and Holder. ${ }^{2}$ This mixing rule is recommended to correlate solubility values obtained at different temperatures since it unifies the overall data handing in only one set of parameters. Therefore the solubility in supercritical $\mathrm{CO}_{2}$ would be tested with other compounds dissolved in coffee oil or even other oils using the mixing rule of Mohamed and Holder. ${ }^{2}$

\section{ACKNOWLEDGEMENTS}

The authors thank Fundação de Amparo à Pesquisa do Estado de São Paulo (FAPESP - grant 02/07753-0) and Conselho Nacional de Desenvolvimento Científico e Tecnológico (CNPq - fellowships) for financial support.

\section{REFERENCES}

1. Peng, D.-Y.; Robinson, D. B.; Ind. Eng. Chem. Fundam. 1976, 15, 59.

2. Mohamed, R.S.; Holder, G.D.; Fluid Phase Equilib. 1987, 32, 295.

3. Peker, H.; Srinivasan, M. P.; Smith, J. M.; McCoy, B. J.; AIChE J. 1992, 38,761 .

4. Chang, C. M. J.; Chiu, K. L.; Chen, Y. L.; Chang, C. Y.; Food Chem. 2000, 68, 109.

5. Kopcak, U.; Mohamed, R. S.; J. J. Supercrit. Fluids 2005, 34, 209.

6. Iwai, Y.; Nagano, H.; Lee, G. S.; Uno, M.; Arai, Y.; J. Supercrit. Fluids 2006, 38, 312 .

7. Saldaña, M. D. A.; Zetzl, C.; Mohamed, R. S.; J. Agric. Food Chem. 2002a, 50, 4820.

8. Saldaña, M. D. A.; Mazzafera, P.; Mohamed, R. S.; Fluid Phase Equil. 2002b, 194, 885 .

9. Johannsen, M.; Brunner, G.; Fluid Phase Equilib. 1994, 95, 216.

10. Mchugh, M. A.; Krukonis, V. J.; Supercritical fluid extraction: principles and practice. Butterworths Publishers: Boston, 1994.

11. Ting, S. S. T.; Mcnaughton, S. J.; Tomasko, D. L.; Foster, N. R.; Ind. Eng. Chem. Res. 1993b, 32, 1471.

12. Bothe, H.; Cammenga, H.K.; J. Thermal Anal. 1979, 16, 267.

13. Morita, A.; Kajimoto, O.; J. Phys. Chem. 1990, 94, 6420.

14. Bai, S.; Craig, M. V.; Liu, F.; Mayne, C. L.; Pugmire, R.J.; Grant, D. M.; J. Phys. Chem. 1997, 101, 2923.

15. Bulgarevicg, D. S.; Sako, T.; Sujeta, T.; Otake, K.; Takebayashi, Y.; Kamizawa, C.; Horikawa, Y.; Kato. M.; Ind. Eng. Chem. Res. 2002, 41, 2074.

16. Chimowitz, E. H.; Kelley, F. D.; Munoz, F. M.; Fluid Phase Equilib. 1988, 44, 23

17. Johnston, K. P.; Barry, S. E.; Read, N. K.; Holcomb, T. R.; Ind. Eng. Chem. Res. 1987, 26, 2372.

18. Temelli, F.; J. Food Sci. 1992, 57, 440.

19. Azevedo, A. B. A.; Mohamed, R. S.; Kopcack, U.; J. Supercrit. Fluids 2003, 27, 223.

20. Azevedo, A. B. A.; Tese de Doutorado, Universidade Estadual de Campinas, Brasil, 2005.

21. Nilsson, W. B.; Hudson, J.K.; JAOCS 1993, 70, 749.

22. Neves, G. B. M.; Dissertação de Mestrado, Universidade Estadual de Campinas, Brasil, 1996.

23. Ting, S. S. T.; Tomasko, D. L.; Macnaughton, S. J.; Foster, N.R.; Ind. Eng. Chem. Res. 1993a, 32, 1482.

24. Brunner, G.; Gas Extraction an introduction to fundamentals of supercritical fluids and the applications to separation processes, Steinkopff Darmstadt Springer: New York, 1994.

25. Vasapollo, G.; Longo, L.; Rescio, L.; Ciurlia, L.; J. Supercrit. Fluids 2004, 29, 87.

26. Schaeffer, S. T.; Zalkow, L. H.; Teja, A. S.; AIChE 1988, 34, 1740. 